\title{
Investigating the nature of chiral near-field interactions
}

\author{
Lauren E. Barr," Simon A. R. Horsley, Ian R. Hooper, Jake K. Eager, Cameron P. Gallagher, Samuel M. Hornett, \\ Alastair P. Hibbins, and Euan Hendry \\ Department of Physics and Astronomy, University of Exeter, Exeter EX4 4QL, United Kingdom
}

(Received 6 November 2017; published 18 April 2018)

\begin{abstract}
In recent years, there have been reports of enhanced chiroptical interactions in the near-fields of antennas, postulated to be mediated by high spatial gradients in the electromagnetic fields. Here, using gigahertz experimentation, we investigate the nature of the chiral near-field generated by an array of staggered-rod antennas through its interaction with an array of aligned, subwavelength metallic helices. This allows us to eliminate many potential origins of enhancements, such as those associated with plasmon-exciton interactions, and search solely for enhancements due to the high spatial gradients in the chirality of the fields around chiral antennas (so-called 'superchiral fields'). By comparing the strength of the chiral interaction with our helices to that of a homogeneous chiral layer with effective material parameters, we find that the strength of this chiral interaction can be predicted using a completely local effective medium approximation. This suggests no obvious enhancement in the chiral interaction in the near-field and indicates that nonlocal interactions are negligible in this system.
\end{abstract}

DOI: 10.1103/PhysRevB.97.155418

\section{INTRODUCTION}

Throughout nature we find objects and systems that are distinguishable from their reflection in a mirror: from simple amino acids to the complex proteins they constitute. These objects are chiral and can exist in two states (enantiomers) of opposite handedness, where one enantiomer is the mirror image of the other. Handedness is fundamental in determining how a molecule will interact with its environment, and there are numerous examples of biologically inert or beneficial molecules with an 'evil twin' [1]. The ability to distinguish different enantiomers is therefore of paramount importance, for example, for safe and efficient drug production.

One of the few ways to distinguish enantiomers is through their differing chiroptical interactions with chiral electromagnetic (EM) fields, such as in circular dichrosim. This problem becomes particularly interesting when we step away from the dipolar regime, i.e., when we can no longer assume that molecules are subwavelength and act as dipoles under planewave excitation. Several recently published papers report a large enhancement (up to 6 orders of magnitude) in the strength of chiroptical interactions involving biomolecules on chiral plasmonic structures [2-5] and in the nodes of chiral standing waves [6]. It has been proposed that these strengthened interactions are mediated by 'superchiral' fields and allow more sensitive detection and efficient selection of chiral molecules and even indicate their hierarchical structure [7]. However, the nature and origin of this enhancement remain difficult to decipher $[3,8,9]$ because many factors come into play in these complex systems, and spin and orbital angular momenta are not well defined [10-12]. There are also several measures of chirality that may be employed [13-15], further complicating analysis.

*1b499@exeter.ac.uk
One proposed interpretation of the observed chiral enhancement in the vicinity of nanoparticles has its origins in multipolar contributions to the chiroptical interaction [2], originating from spatial gradients in the electric field [16]. Normally multipolar contributions can be ignored, as they are origin dependent and average to 0 within the random dipole approximation (where the constituent molecules are small and randomly oriented). However, for a surface composed of resonant plasmonic antennas, which give rise to very large spatial gradients in the fields, the contribution from such terms to the strength of a chiral interaction with adsorbed molecules remains unclear [17-20].

In this paper we study how chiral EM near-fields interact with helical 'molecules.' The chiral fields are generated by illuminating a staggered pair of rod antennas with gigahertz radiation [21], while very subwavelength metallic helices act as the molecules. It is thought that multipolar, nonlocal contributions can be significant when the EM field changes on a length scale comparable to the size of the chiral element $[22,23]$, as is the case in our strongly evanescent chiral nearfields. Our experiment is designed to test whether contributions to the chiral interactions exist that are not predicted by models that assume an effective homogeneous chiral medium, i.e., one where there are no discrete chiral elements of a size comparable to the wavelength scale. However, we discover that the strength of this chiral interaction can be predicted using an effective medium approximation, describing the helical layer with wave-vector-independent permittivity, permeability, and chirality material parameters. This indicates that multipolar chiral effects do not contribute significantly to the chiroptical interaction in the near-field.

\section{THE STAGGERED-ROD ANTENNA ARRAY}

Throughout this work we follow the definition of EM chirality introduced by Tang and Cohen [24]. They define 
the chirality of a time-varying EM field as the time-even pseudoscalar,

$$
C=2 \omega \mathbb{I}\left(\tilde{E}^{*} \cdot \tilde{B}\right),
$$

where $\tilde{E}$ and $\tilde{B}$ are complex electric and magnetic fields, respectively, and $\omega$ is the angular frequency. In other words, the chirality of a field is proportional to the product of parallel components of the electric and magnetic fields that have $\mathrm{a} \pm \frac{\pi}{2}$ phase difference. The chirality of a circularly polarized wave, assuming unit intensity, is therefore $C= \pm 2 \omega / c$, where the \pm correspond to right- and left-handed waves. We use this definition to design antennas that generate chiral fields on resonance, following [21].

Consider the fundamental resonance of a half-wavelength rod antenna: on resonance, a current maximum (and corresponding maximum in magnetic field) is found at its midlength, while the electric field is concentrated at both ends of the rod. Though rod antennas do not possess chiral symmetry by themselves, one can arrange them in two-dimensional chiral ensembles by staggering them [21]. To obtain a strong overlap between the electric- and the magnetic-field regions, they can be arranged in pairs as shown in Fig. 1(a): for this arrangement, and because of the fixed $\left( \pm \frac{\pi}{2}\right)$ phase relationship between electric and magnetic fields of the eigenmodes of the staggered geometry, a chiral EM field is generated at the center of the unit cell. The nature of this field differs from that of circularly polarized waves, in that the EM chirality decays quickly in space. This is illustrated in Fig. 1(a), which shows the chiral density of the EM field above and below one unit cell of the array as calculated using Eq. (1). This large spatial gradient in the field, in principle, should allow coupling to multipolar modes of an object in the vicinity of the field, as it contains high wave-vector components.

Figure 1(b) shows the transmission spectrum of the staggered-rod array: both experimental data and the results of a finite-element method (FEM) model (COMSOL Multiphysics). (Note that the frequency range of the presented data is limited by the parameter extraction technique, explained below.) A resonant dip in the intensity of transmission at $16 \mathrm{GHz}$, where the wavelength is roughly equal to twice the length of one rod, is evident when the polarization of the incident electric field is parallel to the major rod axis. At this resonance the fields around the antennas satisfy the definition in (1) and the chirality of the near-fields is strong. Hence it is in this region (and below, as the resonance shifts to lower frequencies upon placing a chiral material next to the antenna array) that we focus most of our attention. The circular transmission coefficients $\tau$ can be calculated from the four complex linear transmission coefficients $t$, using the equations

$$
\begin{aligned}
& \tau_{ \pm \pm}=\frac{t_{x x}+t_{y y} \pm i\left(t_{x y}+t_{y x}\right)}{2}, \\
& \tau_{ \pm \mp}=\frac{t_{x x}-t_{y y} \mp i\left(t_{x y}-t_{y x}\right)}{2} .
\end{aligned}
$$

The first subscripts on $\tau$ and $t$ denote incident polarization [right-handed (+) and left-handed (-) circular polarizations, or $x$ and $y$ linear polarizations]; the second subscript is the detected polarization. These are used in the calculation of circular dichroism (CD). Since circular polarization conversion
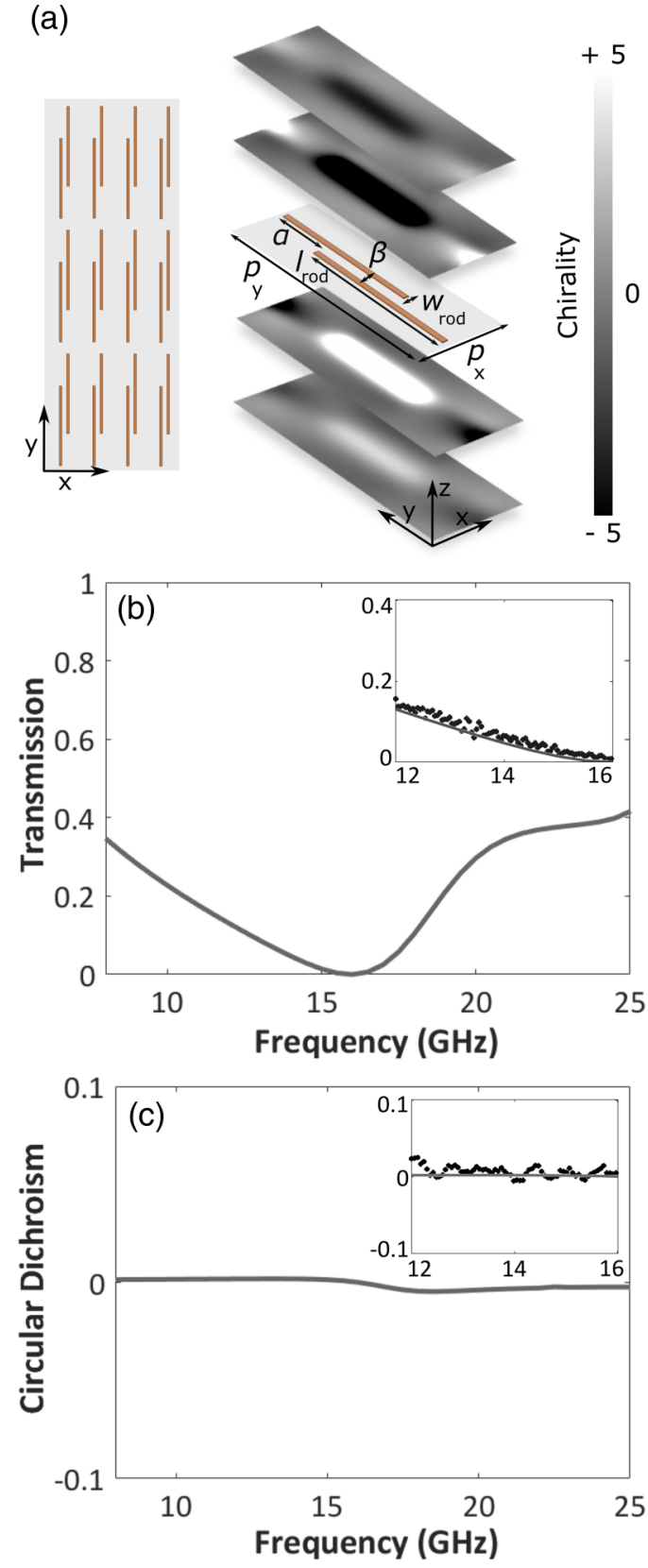

FIG. 1. (a) Schematic of the staggered-rod antenna array. The surface plots at the right illustrate the chirality of the modeled fields 0.4 and $0.8 \mathrm{~mm}$ above and below a unit cell at the resonant frequency of $16 \mathrm{GHz}$, normalized to a circularly polarized plane wave. The dimensions are $\alpha=2.6 \mathrm{~mm}, \beta=300 \mu \mathrm{m}, p_{x}=3 \mathrm{~mm}$, $p_{y}=9.1 \mathrm{~mm}, l_{\text {rod }}=6.4 \mathrm{~mm}$, and $w_{\text {rod }}=200 \mu \mathrm{m}$; the rods have a thickness of $\beta=35 \mu \mathrm{m}$. (b) Finite-element-method (FEM) model predictions of the transmission of linearly polarized radiation through the staggered-rod array, with polarization along the rod axis ( $y$ polarized). Inset: Comparison between experimental (circles) and simulated (line) transmission in the frequency region of interest. (c) FEM predictions of the circular dichroism (CD) of the staggered-rod array. Inset: Comparison between experimental (circles) and predicted (line) $\mathrm{CD}$ in the region of interest.

is a signature of anisotropy, not chirality, we ignore these contributions and consider only the conserved circular polarization 
components in the calculation:

$$
\mathrm{CD}=\frac{\left|\tau_{++}\right|^{2}-\left|\tau_{--}\right|^{2}}{\left|\tau_{++}\right|^{2}+\left|\tau_{--}\right|^{2}} .
$$

Figure 1(c) shows the CD of the staggered-rod antenna array obtained from both the FEM model and the experimental measurements. With the extremely subwavelength thickness $(35 \mu \mathrm{m})$ of the antenna array, its CD is small but nonzero due to the presence of the substrate, which breaks the out-of-plane symmetry.

\section{THE HELICAL MATERIAL}

To probe the interaction of the chiral near-fields generated by the array of staggered-rod antennas, we use a twodimensional rectangular array of steel helices. The helices each have three turns, and dimensions [illustrated in Fig. 2(a)] such that they are subwavelength across the investigated frequency range and can be considered to be perfectly electrically conducting. The pitch of the array was also chosen to mismatch that of the staggered-rod antenna array, so that on average the relative positions of individual helices and antennas will not contribute to the measurements; i.e., we eliminate any origin-dependent effects. The major axes of all the helices are aligned in the same direction, making the array anisotropic as well as chiral, hence ensuring that we are able to probe any contributions to the chirality of the interaction from spatial gradients in the near-fields. The helices are placed into an array of rectangular-cross-section indentations in an additive-layer printed dielectric plate (relative permittivity of $2.73+i 0.27$ across the frequency band of interest, determined from stripline measurements). A thin layer of paraffin wax was used to secure the helices in place.

We now study the EM response of the helix array (plus dielectric plate) in isolation: its transmission and $\mathrm{CD}$ are measured using the approach described for the staggered-rod antennas [Eqs. (2)-(4)] and are shown in Figs, 2(b) and 2(c). When the incident radiation is polarized along the major axis of the helices, the resonant dip in transmission occurs for a wavelength equal to roughly twice the length of the wire in the helix. Importantly the CD of the helix array in the frequency range where the staggered rods are resonant is relatively small, shown in the inset in Fig. 2(c). Working below the fundamental resonance of the helical material is reminiscent of typical biosensing experiments [1] and provides the opportunity to enhance the $\mathrm{CD}$ in a spectral region where it is not already large.

Since the helices in the layer are very subwavelength, one might assume that a homogenized description of the helix array may be sufficient. However, the effective medium approximation, by definition, assumes that the elements comprising the material are infinitely small compared to the wavelength, hence this model will not encompass any multipolar contributions to the chiral interaction. It should be noted that, as there is only a single layer of helices, the system is best described as a metasurface, rather than bulk metamaterial. This is evident because the value of extracted parameters will change with the addition of extra layers. However, an effective medium description of a metasurface of this kind has proven useful in various other systems [25,26]. Such an effective medium is
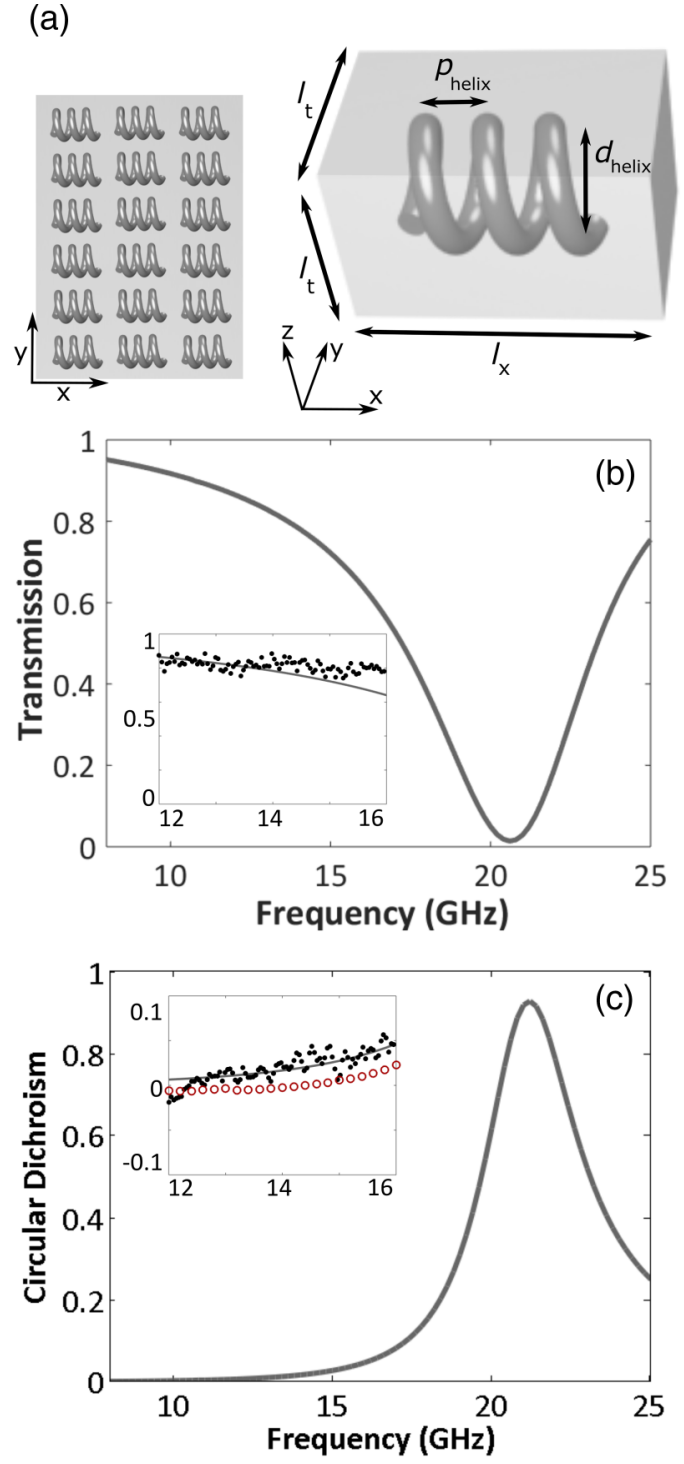

FIG. 2. (a) Schematic of the helical layer with an enlargement of one unit cell. The dimensions are $p_{\text {helix }}=0.4 \mathrm{~mm}, d_{\text {helix }}=0.72 \mathrm{~mm}$, $l_{x}=2.0 \mathrm{~mm}$, and $l_{\mathrm{t}}=1.4 \mathrm{~mm}$. (b) Simulated transmission of linearly polarized radiation through this layer with the polarization along the helix axis ( $x$ polarized). Inset: Comparison between experimental (circles) and simulated (line) transmission in the frequency region of interest, i.e., around the resonance of the staggered-rod array [Fig. 1(b)], and reliable parameter extraction. (c) Simulated circular dichroism for this layer. Inset: Comparison between circular dichroism of the array of helices extracted from a full FEM model with a helix unit cell (line) using the unit cell in (a), a FEM model using an effective medium description of the array (open red circles), and experimental data (filled black circles).

described by a tensorial permittivity, $\epsilon$, permeability, $\mu$, and chirality, $\kappa$, which characterize the electromagnetic response of the system under plane-wave excitation. The constitutive relations for a such a material are

$$
\bar{D}=\overline{\bar{\epsilon}} \cdot \bar{E}+\overline{\bar{\xi}} \cdot \bar{H}
$$

and

$$
\bar{B}=\overline{\bar{\zeta}} \cdot \bar{E}+\overline{\bar{\mu}} \cdot \bar{H},
$$


where $\bar{D}$ and $\bar{B}$ are the electric displacement and magnetization of the material, $\bar{E}$ and $\bar{H}$ are the electric and magnetic fields, $\overline{\bar{\epsilon}}$ and $\overline{\bar{\mu}}$ are the permittivity and permeability of the material, and $\overline{\bar{\zeta}}=-\bar{\xi}$ are the cross-coupling material parameters. Throughout the analysis we assume an $e^{i \omega t}$ time dependence to the waves. In general the material parameters are $3 \times 3$ tensors that may have off-diagonal components. However, as we assume that the material is uniaxially anisotropic, the permittivity and permeability take the forms

$$
\overline{\bar{\epsilon}}=\left(\begin{array}{ccc}
\epsilon_{\mathrm{x}} \epsilon_{0} & 0 & 0 \\
0 & \epsilon_{\mathrm{t}} \epsilon_{0} & 0 \\
0 & 0 & \epsilon_{\mathrm{t}} \epsilon_{0}
\end{array}\right)
$$

and

$$
\overline{\bar{\mu}}=\left(\begin{array}{ccc}
\mu_{x} \mu_{0} & 0 & 0 \\
0 & \mu_{t} \mu_{0} & 0 \\
0 & 0 & \mu_{t} \mu_{0}
\end{array}\right),
$$

where the subscript $t$ denotes the direction transverse to the major helix axis, equivalent to both $y$ and $z . \epsilon_{0}$ and $\mu_{0}$ are the permittivity and permeability of the free space, respectively. A further simplification we make is that the material only has chirality, $\kappa$, along one direction (the major helix axis, $x$ ), and so the cross-coupling parameters have the form

$$
\bar{\xi}=\left(\begin{array}{ccc}
-i \kappa \sqrt{\mu_{0} \epsilon_{0}} & 0 & 0 \\
0 & 0 & 0 \\
0 & 0 & 0
\end{array}\right)
$$

and

$$
\overline{\bar{\zeta}}=\left(\begin{array}{ccc}
i \kappa \sqrt{\mu_{0} \epsilon_{0}} & 0 & 0 \\
0 & 0 & 0 \\
0 & 0 & 0
\end{array}\right) .
$$

We ignore the small chiral response perpendicular to the helix axis, which is due to the finite number of turns in each helix. An analytical description of the complex reflection and transmission coefficients through an anisotropic chiral material can be derived by substituting the above constitutive relations into the wave equation and applying appropriate boundary conditions to the electric and magnetic fields, following [27]. More details on the calculations are given in Appendix C. The homogenized parameters describing our chiral material are derived by fitting the complex reflection and transmission spectra predicted from a full FEM model to these analytical predictions. The frequency-dependent extracted parameters are plotted in Appendix C, along with an outline of the extraction technique and limitations. It should be noted that this extraction technique will only yield reliable results at frequencies away from the helix resonance, where the layer has a subwavelength thickness, in the frequency range where the staggered-rod array is resonant. Hence the spectra are only reported to a maximum frequency of $16 \mathrm{GHz}$. The inset in Fig. 2(c) shows the $\mathrm{CD}$ of the effective (i.e., homogenized) medium (open red circles) compared to the experimental results and full (helix unit-cell) FEM model.

\section{PROBING THE CHIRAL NEAR-FIELDS}

We now consider the response of the combined system: the helical layer placed on one face of the rod antenna array. To
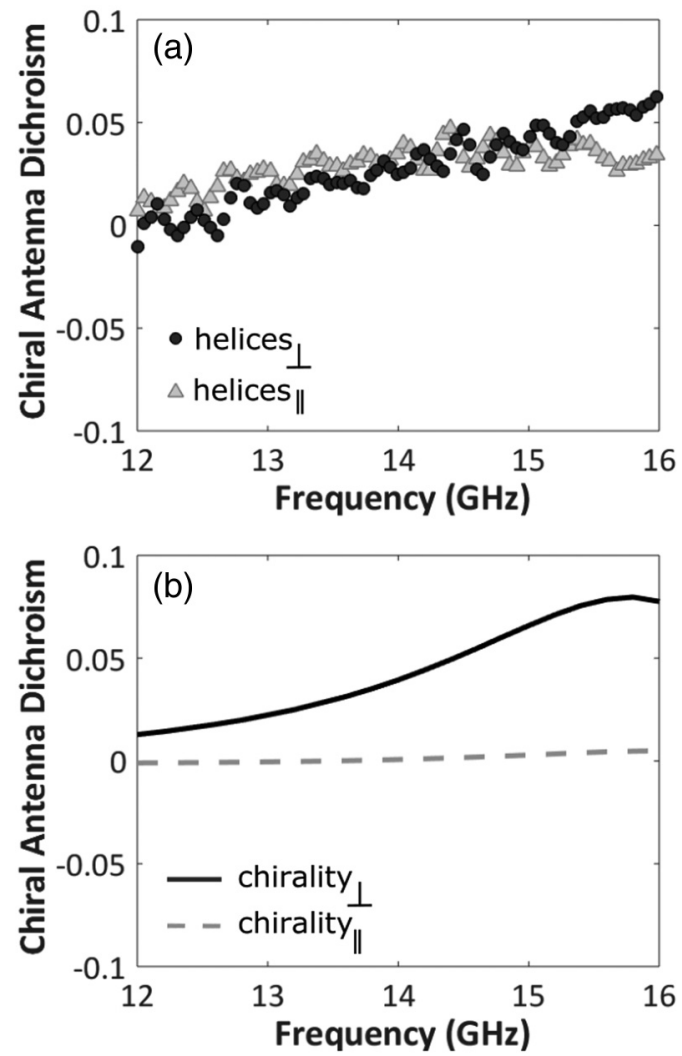

FIG. 3. (a) Experimental chiral antenna dichroism (CAD) of the helix layer, for helices oriented parallel (gray triangles) and perpendicular (black circles) to the major rod axis. (b) Simulated (FEM) $\mathrm{CAD}$ using an effective medium representation of the helical array with the chirality material parameter, $\kappa$, oriented parallel (dashed gray line) and perpendicular (solid black line) to the major rod axis.

define the strength of interaction between the chiral fields generated by the staggered-rod antennas and the helical layer, it is necessary to introduce a new quantity: chiral antenna dichroism (CAD) [2]. As a near-field analogy of circular dichroism, where field handedness is generated by the symmetry of the antenna, $\mathrm{CAD}$ is a measure of the difference in intensity of the radiation transmitted through the combined system when the handedness of the antenna (staggered-rod array) is switched, normalized to the sum of the two measurements:

$$
\mathrm{CAD}=\frac{\left|\tau_{\mathrm{LH}}\right|^{2}-\left|\tau_{\mathrm{RH}}\right|^{2}}{\left|\tau_{\mathrm{LH}}\right|^{2}+\left|\tau_{\mathrm{RH}}\right|^{2}},
$$

where the subscript on $\tau$ represents the handedness of the antenna array (as seen from the side closest to the helices). We expect a nonzero value of CAD only when a chiral material is within the near-fields of the antennas, indicating a difference in coupling between RH and LH antennas. Figure 3(a) shows the experimentally measured $\mathrm{CAD}$ when the helices are parallel and perpendicular to the major axis of the rods. It is clear that, since the CAD is nonzero, we are able to probe the chiral interaction between the antenna array and the helical array in a manner similar to that in [2].

At this point, we consider the nature of this chiral interaction. It is interesting to note that the CAD [Fig. 3(a)] of the helical array is of a similar magnitude to its circular dichroism 
[Fig. 2(c)] in the same frequency range. This suggests that any enhancement in the interaction due to the presence of rapidly decaying near-fields is too small to contribute significantly to the measurement. Furthermore, the measured CAD of helices oriented parallel and perpendicular to the rod axis are very similar. This indicates that the helical array is not especially sensitive to the spatial variation of the chiral near-fields around the staggered-rod antennas.

It is therefore prudent to question whether we observe any evidence of nonlocality in the chiral interaction, i.e., whether the spatial gradients allow coupling to multipolar modes and whether this affects the observed CAD. If a nonlocal contribution is significant, a completely local description of the helical material, as described above, would be inadequate to describe the interaction. Instead, we would need to adopt a nonlocal description of the helical metamaterial, where the material parameters are a function of both the frequency and the wave vector. To determine if this is indeed the case, in Fig. 3(b) we present the CAD calculated from a FEM model with an effective medium, described by $\epsilon, \mu$, and $\kappa$, substituted for the array of helices. Results are shown for the chiral material parameter, $\kappa$, oriented both parallel and perpendicular to the major rod axis. (Note that we do not expect good agreement with experiment [Fig. 3(a)] in the parallel chirality case, due to approximations made in the analytical description [27].) The pivotal point is that the orders-of-magnitude enhancements predicted in previous works [2,3,7] are not observed here. Considering the approximations used in the model, it is remarkable that, although the exact frequency dependence of the spectra are not perfectly replicated, the sign and magnitude closely resemble those of the experimentally measured CAD. This suggests that nonlocal effects and multipolar enhancements do not contribute significantly to our CAD measurement; the array of helices is essentially behaving as a local material. Indeed, it is clear that the contribution to the chiral interaction from multipolar components in the field is minimal.

\section{SEPARATION DEPENDENCE OF CHIRAL ANTENNA DICHROISM}

To further understand the interaction between the helices and the evanescently decaying fields close to the staggered-rod array, a study of the separation dependence of the CAD was also carried out. The experimental results in Fig. 4(a) show a slightly counterintuitive result. If we were to assume that the CAD is a near-field effect, we would predict that as the helices are moved out of the near-fields the CAD should decrease exponentially. However, we see that the CAD actually increases with increasing separation. This can be explained as a Drexhage-like effect [28]. When the helical material, comprised of discrete scatterers, is close to the rod array (which acts like a poor mirror), image currents and image polarizations in the mirror act to cancel the field scattered by the helices [29]. These 'image' helices are of the opposite handedness to the helices in the real helical material, so it is no surprise that the CAD is small when the helices and the rod array are close to each other [30]. As the separation is increased, but is still less than one wavelength, the image helices become weaker and the CAD increases. This effect is also seen when the electric-field intensity is measured just above the rod array using a dipole antenna. If the sepa-
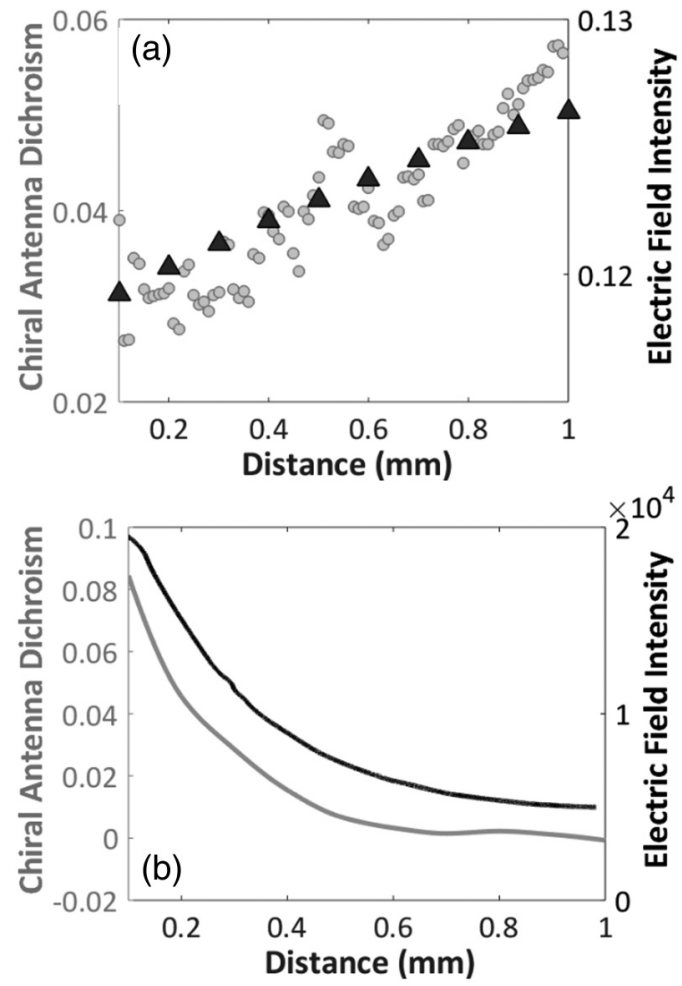

FIG. 4. (a) Experimentally measured chiral antenna dichroism (gray circles, corresponding to left $y$ axis) as a function of the separation, for helices perpendicular to the major rod axis at $16 \mathrm{GHz}$. Black triangles (corresponding to right $y$ axis) show the electric-field intensity measured by a small dipole antenna as a function of its distance from the rod array, also at $16 \mathrm{GHz}$. (b) Simulated chiral antenna dichroism of the effective medium (gray line, corresponding to left $y$ axis) at $16 \mathrm{GHz}$ as a function of the separation; electric-field intensity (black line, corresponding to right $y$ axis) extracted from a FEM model from the center of a staggered-rod unit cell, as a function of the distance from the rod array at $16 \mathrm{GHz}$.

ration is increased beyond one wavelength, the characteristic oscillations of the Drexhage effect are also observed.

However, this effect is not seen in the case of the effective medium model, as here there are no discrete scatterers, which are necessary to observe the Drexhage effect. Therefore we see a decay of the CAD with increasing separation. This decay follows well the decay of the electric-field intensity away from the center of one unit cell in the antenna array, extracted from the FEM model, as plotted in Fig. 4(b).

From these data we can draw the conclusion that the presence of discrete chiral elements in the helical array does influence the CAD as a function of the separation between the helical material and the antenna array. Still, no significant enhancement is present over the effective medium approximation. It also highlights the fact that interactions between chiral materials and structured surfaces are more complicated than is apparent at first glance, and the full details of the mechanisms behind such interactions have yet to be fully understood.

\section{CONCLUSION}

In this paper, the chiral EM near-fields generated by an array of staggered-rod antennas were investigated using an array 
of metallic helices as a probe. The chiral antenna dichroism (CAD) was used as a measure of the interaction strength between the chiral near-fields and the helices. This was measured experimentally and compared to a model of an equivalent homogeneous medium. It was found that the CAD of the helical array could be predicted fairly accurately by approximating it as a homogeneous chiral material. It is therefore clear that the nonlocal contribution to the chiral interaction is minimal.

However, there are a number of important differences that may account for discrepancies between our experiments and those in [2]. First, the frequency of radiation used in this report is much lower than that reported in [2]. As a result, the contribution to the interaction from the plasmonic behavior of the antennas is not included. It is well accepted that the concentrated electric fields around plasmonic particles can increase the excitation rate of molecules, increasing the sensitivity with which they are detected in general [31]. Recent works have also claimed large enhancements in the circular dichroism measured from chiral molecules placed on achiral plasmonic structures [32], [33], proposing that the enhanced circular dichroism is due to strong absorption in the metal at the plasmon frequency [34]. In such circumstances the plasmonexciton interaction is key, and the spatial structure of chiral evanescent fields around the particles themselves is likely to play little, if any, role. Another important factor may be the alignment of molecules adsorbed on a surface in [2]. The orientation of molecules with respect to the electric-field vectors in the vicinity of the plasmonic particles has been shown to play an important role in the magnitude of CD signals in [35] and, also, suggested in [36] and [37]. Once again this effect seems to rely on plasmonic enhancement through a Purcell-type effect, and not directly on the spatial structure of chiral evanescent fields. In any respect, it is explicit that the origin of the signal enhancement in [2] demands further investigation.

\section{DATA AVAILABILITY}

All data created during this research are openly available from the University of Exeter's institutional repository in Ref. [38].

\section{ACKNOWLEDGMENTS}

L.E.B., I.R.H., C.P.G., and A.P.H. wish to acknowledge financial support form the Engineering and Physical Sciences Research Council (EPSRC) of the United Kingdom, via the EPSRC Centre for Doctoral Training in Metamaterials (Grant No. EP/L015331/1). C.P.G. also wishes to acknowledge support from Defense Science and Technology Laboratory (DSTL). S.A.R.H. acknowledges financial support from the Royal Society and TATA (RPG-2016-186). E.H. and S.M.H. wish to acknowledge support from EPSRC fellowship EP/K041215/1.

\section{APPENDIX A: FABRICATION}

The staggered-rod antennas were manufactured using a lithographic technique; 1.6-mm-thick printed circuit boards with a $35-\mu \mathrm{m}$-thick layer of copper and a positive resist were exposed under a UV lamp with an appropriate mask, then developed and etched in ferric chloride to produce an array of copper rods. Two of these were created, one of each handedness, as the presence of the FR4 substrate prevented the use of both sides of the one piece. The final arrays were $260 \times 260 \mathrm{~mm}$ in size and contained $54 \times 9$ unit cells in a rectangular lattice.

The helices, manufactured by Huidong Linglong Spring Co., Ltd., were placed within a dielectric grid made using a selective laser sintering technique from 3D Systems On Demand Manufacturing, QuickParts. No attempt was made to azimuthally align the helices. A thin layer of paraffin wax (permittivity, $2.2+i 0.0044$ ) was poured on top to secure the helices in place, then polished to a smooth surface.

\section{APPENDIX B: MEASUREMENT}

The experimental measure of the transmission was obtained by placing the sample between two collimating mirrors, with standard-gain horn antennas at the focus of each of the mirrors, both of which are connected to a vector network analyzer (Anritsu MS4644A). One horn antenna emits linearly polarized microwave radiation, which, upon reflection from the mirror, becomes a near-collimated beam with approximately planar wave fronts. The second horn antenna collects the refocused radiation after it has passed through the sample and been reflected from the second collimating mirror. All measurements are carried out at normal incidence. Each of the horn antennas can be azimuthally rotated by $90^{\circ}$, allowing the collection of four polarization-dependent, complex $S$ parameters.

\section{APPENDIX C: PARAMETER EXTRACTION}

\section{Analytic reflection and transmission coefficients}

In the text, a material consisting of a single layer of aligned metallic helices embedded in a dielectric host is described using an effective parameter approximation. The resultant homogenized material is uniaxially anisotropic and chiral, with the chirality acting only along one direction (the major axis of the helices, $x$ ). The effective material parameters are extracted by fitting analytical complex reflection and transmission coefficients to those obtained from a FEM model. These analytical reflection and transmission coefficients are found by deriving the wave equation in a material with constitutive relations as given in Eqs. (5) and (6) in the text from the standard Maxwell's equations and eliminating $\bar{D}, \bar{B}$, and $\bar{H}$ :

$$
\begin{aligned}
& \Delta \times\left(\overline{\bar{\mu}}^{-1} \cdot \Delta \times \bar{E}\right)+i \omega\left[\Delta \times\left(\overline{\bar{\mu}}^{-1} \cdot \overline{\bar{\xi}} \cdot \bar{E}\right)-\overline{\bar{\zeta}} \cdot \overline{\bar{\mu}}^{-1}\right. \\
& \cdot(\Delta \times \bar{E})]+\omega^{2}\left(\overline{\bar{\zeta}} \cdot \overline{\bar{\mu}}^{-1} \cdot \overline{\bar{\xi}} \cdot \bar{E}-\overline{\bar{\epsilon}} \cdot \bar{E}\right)=0 .
\end{aligned}
$$

Solving this wave equation for the case of a plane wave propagating in the $+z$ direction we find the wave numbers of the two elliptically polarized modes for the material:

$$
k_{1,2}^{2}=\omega^{2}\left[\frac{\epsilon_{\mathrm{x}} \mu_{\mathrm{t}}+\epsilon_{\mathrm{t}} \mu_{\mathrm{x}}}{2} \pm \sqrt{\left(\frac{\epsilon_{\mathrm{x}} \mu_{\mathrm{t}}-\epsilon_{\mathrm{t}} \mu_{\mathrm{x}}}{2}\right)^{2}+\epsilon_{\mathrm{t}} \mu_{\mathrm{t}} \zeta \xi}\right] .
$$

Considering a plane wave normally incident on the material from the $-z$ direction we define the incident, reflected, and 
transmitted electric fields and use these to find the reflection and transmission coefficients for waves co- and cross-polarized to the incident wave polarization:

$$
\begin{aligned}
& R_{\mathrm{co}}=\frac{E_{\mathrm{co}}^{r} e^{-i k_{\mathrm{d}} z_{\text {in }}}}{E_{0} e^{i k_{\mathrm{d}} z_{\mathrm{in}}}}, \\
& R_{\mathrm{cr}}=\frac{E_{\mathrm{cr}}^{r} e^{-i k_{\mathrm{d}} z_{\text {in }}}}{E_{0} e^{i k_{\mathrm{d}} z_{\mathrm{in}}}}, \\
& T_{\mathrm{co}}=\frac{E_{\mathrm{co}}^{t} e^{i k_{\mathrm{d}} z_{\mathrm{out}}}}{E_{0} e^{i k_{\mathrm{d}} z_{\text {in }}}},
\end{aligned}
$$

$$
T_{\mathrm{cr}}=\frac{E_{\mathrm{cr}}^{t} e^{i k_{\mathrm{d}} z_{\mathrm{out}}}}{E_{0} e^{i k_{\mathrm{d}} z_{\mathrm{in}}}},
$$

where $k_{d}=\omega \sqrt{\mu_{\mathrm{d}} \epsilon_{\mathrm{d}}}$ is the wave vector in the dielectric medium surrounding the helices, $z_{\text {in }}$ is the plane of the material where the plane wave is incident, and $z_{\text {out }}$ is the plane of the material through which the wave is transmitted.

By matching the tangential $E$ and $H$ fields at the two boundaries of the material we arrive at an analytical description of the reflection and transmission coefficients in terms of the material parameters,

$$
\left[\begin{array}{l}
R_{\mathrm{co}} \\
R_{\mathrm{cr}} \\
T_{\mathrm{co}} \\
T_{\mathrm{cr}}
\end{array}\right]=\left(\overline{\bar{N}}-\overline{\bar{M}}_{2} \cdot M_{1}^{-1} \cdot \overline{\bar{N}}\right)^{-1} \cdot\left(\bar{W}+\overline{\bar{M}}_{2} \cdot M_{1}^{-1} \cdot \bar{W}\right)
$$

where the matrices are

$$
\begin{aligned}
& \overline{\bar{N}}=\left[\begin{array}{cccc}
\sin \phi & -\cos \phi & 0 & 0 \\
\cos \phi & \sin \phi & 0 & 0 \\
0 & 0 & \sin \phi & \cos \phi \\
0 & 0 & -\cos \phi & \sin \phi
\end{array}\right] \\
& \bar{W}=\left[\begin{array}{c}
\sin \phi \\
\cos \phi \\
0 \\
0
\end{array}\right] \\
& \overline{\bar{M}}_{1}=\left[\begin{array}{cccc}
\frac{-i k_{\mathrm{t}}^{2} k_{0} \kappa}{k_{1}} & \frac{i k_{\mathrm{t}}^{2} k_{0} \kappa}{k_{1}} & \frac{-i k_{\mathrm{t}}^{2} k_{0} \kappa}{k_{2}} & \frac{i k_{\mathrm{t}}^{2} k_{0} \kappa}{k_{2}} \\
k_{\mathrm{x}}^{2}-k_{1}^{2} & k_{\mathrm{x}}^{2}-k_{1}^{2} & k_{\mathrm{x}}^{2}-k_{2}^{2} & k_{\mathrm{x}}^{2}-k_{2}^{2} \\
\frac{-i k_{\mathrm{t}}^{2} k_{0} \kappa e^{-i k_{1} d}}{k_{1}} & \frac{i k_{\mathrm{t}}^{2} k_{0} \kappa e^{i k_{1} d}}{k_{1}} & \frac{-i k_{\mathrm{t}}^{2} k_{0} \kappa e^{-i k_{2} d}}{k_{2}} & \frac{i k_{\mathrm{t}}^{2} k_{0} \kappa e^{i k_{2} d}}{k_{2}} \\
\left(k_{1}^{2}-k_{\mathrm{x}}^{2}\right) e^{-i k_{1} d} & \left(k_{1}^{2}-k_{\mathrm{x}}^{2}\right) e^{i k_{1} d} & \left(k_{2}^{2}-k_{\mathrm{x}}^{2}\right) e^{-i k_{2} d} & \left(k_{2}^{2}-k_{\mathrm{x}}^{2}\right) e^{i k_{2} d}
\end{array}\right],
\end{aligned}
$$

and

$$
\overline{\bar{M}}_{2}=\frac{k_{\mathrm{t}} \eta_{\mathrm{d}}}{\eta_{\mathrm{t}}}\left[\begin{array}{cccc}
i k_{0} \kappa & i k_{0} \kappa & i k_{0} \kappa & i k_{0} \kappa \\
\frac{k_{1}^{2}-k_{\mathrm{x}}^{2}}{k_{1}} & \frac{k_{\mathrm{x}}^{2}-k_{1}^{2}}{k_{1}} & \frac{k_{2}^{2}-k_{\mathrm{x}}^{2}}{k_{2}} & \frac{k_{\mathrm{x}}^{2}-k_{2}^{2}}{k_{2}} \\
-i k_{0} \kappa e^{-i k_{1} d} & -i k_{0} \kappa e^{i k_{1} d} & -i k_{0} \kappa e^{-i k_{2} d} & -i k_{0} \kappa e^{i k_{2} d} \\
\frac{\left(k_{1}^{2}-k_{\mathrm{x}}^{2}\right) e^{-i k_{1} d}}{k_{1}} & \frac{\left(k_{\mathrm{x}}^{2}-k_{1}^{2}\right) e^{i k_{1} d}}{k_{1}} & \frac{\left(k_{2}^{2}-k_{\mathrm{x}}^{2}\right) e^{-i k_{2} d}}{k_{2}} & \frac{\left(k_{\mathrm{x}}^{2}-k_{2}^{2}\right) e^{i k_{2} d}}{k_{2}}
\end{array}\right],
$$

where $\phi$ is the angle of incident polarization, $k_{0}$ is the freespace wave vector of the incident radiation, $k_{1,2}$ are the wave vectors in the material as defined in Eq. (C2), $k_{\mathrm{t}}=\omega \sqrt{\mu_{\mathrm{t}} \epsilon_{\mathrm{t}}}$ is the wave vector transverse to the direction of the chirality, $\eta_{\mathrm{t}}=\sqrt{\frac{\mu_{\mathrm{t}}}{\epsilon_{\mathrm{t}}}}$ is the impedance in this direction, $k_{\mathrm{x}}=\omega \sqrt{\mu_{\mathrm{t}} \epsilon_{\mathrm{x}}}$ is the wave vector along the direction of the chirality, $\eta_{\mathrm{d}}=\sqrt{\frac{\mu_{\mathrm{d}}}{\epsilon_{\mathrm{d}}}}$ is the impedance of the dielectric medium in which the helices are embedded, and $d$ is the thickness of the material. A more complete derivation of these equations can be found in [27].

\section{Fitting and parameter extraction}

Equation (C7) can be used to describe the reflection and transmission through the helical material under plane-wave excitation. The complex material parameters embedded in this equation $\left(\epsilon_{\mathrm{x}}, \epsilon_{\mathrm{t}}, \mu_{\mathrm{x}}, \mu_{\mathrm{t}}\right.$, and $\left.\kappa\right)$ were extracted using data from a finite-element method (FEM) model of an infinite array of metallic (perfectly electrically conducting) helices within a dielectric host. This is in good agreement with the experimentally measured results, as shown in Figs. 2(a) and 2(b).

For this extraction, a nonlinear least-squares algorithm was employed to minimize the difference between co- and crosspolarized reflection and transmission coefficients taken from the FEM model and those calculated from Eq. (C7). This was performed for a single frequency at a time, for four angles of incident polarization $(\phi)$, equally spaced across $2 \pi$ radians, ensuring that the system was not undersampled. By starting at very low frequencies, where the chirality is almost 0 and 

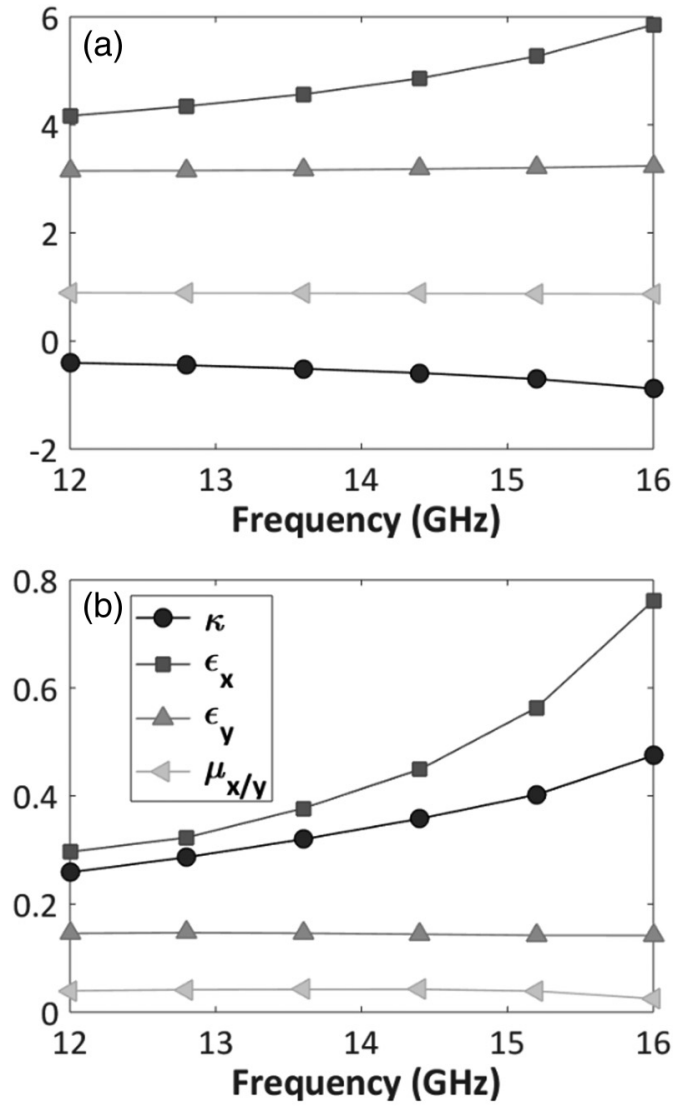

FIG. 5. (a) Real and (b) imaginary parts of the relative material parameters: chirality $(\kappa)$, which acts along the $x$ axis, equivalent to the major helix axis; permittivity $(\epsilon)$, along the $x$ and $y$ axes: and permeability $(\mu)$, which is the same along both the $x$ and the $y$ axis.

the permeability is almost 1 , we were able to use a simplified model to extract only the permittivity and use this as an initial value in the fitting algorithm. The frequency was progressed, and the parameters extracted at the previous frequency were used as the initial guess for the next. In this way the global minimum of the function was found at each frequency.

However, the extraction becomes increasingly difficult as the frequency is further increased towards the resonant frequency of the helical metamaterial. Once the thickness and index of the material become such that more than one wavelength fits inside the layer, the extracted parameters are no longer representative of the material. Therefore, the extracted parameters are presented up to $16 \mathrm{GHz}$ in Fig. 5. It should also be noted that, close to the helix resonance, the meta-atoms are no longer significantly subwavelength, and the reliability of any local effective parameter approximation is greatly reduced. The range covered does include the resonant frequency of the staggered-rod antennas and, so, is acceptable for this work.

\section{APPENDIX D: SIMULATION}

\section{Helix unit cell and antenna array models}

The FEM modeling reported here was performed using COMSOL Multiphysics. Similar models are used for both the staggered-rod antenna array and the helical material. A 'vacuum box' of a height greater than one wavelength is constructed to define a unit cell, with Floquet periodic boundary conditions in the $x$ and $y$ directions to simulate an infinite two-dimensional array. Floquet ports are placed on parallel faces and used to launch and detect linearly polarized plane waves above and below the sample. All metallic elements are modeled using perfect-electric-conductor boundary conditions.

\section{Effective chiral medium model}

When modeling an effective chiral material in COMSOL, simply adjusting the constitutive parameters of the layer is not adequate. It is necessary to adjust the wave equation in one domain of the model to take into account the cross terms in the constitutive relations [Eqs. (5) and (6)]. New definitions of the polarizability are set to take care of the contribution to polarization from the incident magnetic field according to

$$
\begin{gathered}
P_{x}=\epsilon_{0}\left(\epsilon_{x x} E_{x}+\epsilon_{x y} E_{y}+\epsilon_{x z} E_{z}-E_{x}\right)-\frac{i \kappa_{x x} H_{x}}{c}, \\
P_{y}=\epsilon_{0}\left(\epsilon_{y x} E_{x}+\epsilon_{y y} E_{y}+\epsilon_{y z} E_{z}-E_{y}\right)-\frac{i \kappa_{y y} H_{y}}{c}, \\
P_{z}=\epsilon_{0}\left(\epsilon_{z x} E_{x}+\epsilon_{z y} E_{y}+\epsilon_{z z} E_{z}-E_{z}\right)-\frac{i \kappa_{z z} H_{z}}{c} .
\end{gathered}
$$

The magnetization from the incident electric field is included by adjusting the time derivative of the magnetization,

$$
\begin{aligned}
\frac{d H_{x}}{d t} & =\left(\frac{d B_{x}}{d t}+k_{0} \kappa_{x} E_{x}\right)\left(\mu_{0} \mu_{x x}\right)^{-1}, \\
\frac{d H_{y}}{d t} & =\left(\frac{d B_{y}}{d t}+k_{0} \kappa_{y} E_{y}\right)\left(\mu_{0} \mu_{y y}\right)^{-1}, \\
\frac{d H_{z}}{d t} & =\left(\frac{d B_{z}}{d t}+k_{0} \kappa_{z} E_{z}\right)\left(\mu_{0} \mu_{z z}\right)^{-1},
\end{aligned}
$$

and defining the magnetization of the material based on these equations:

$$
\begin{aligned}
& H_{x}=\frac{d H_{x}}{d t}(i \omega)^{-1}, \\
& H_{y}=\frac{d H_{y}}{d t}(i \omega)^{-1}, \\
& H_{z}=\frac{d H_{z}}{d t}(i \omega)^{-1} .
\end{aligned}
$$

In the above equations, $P_{x, y, z}$ and $H_{x, y, z}$ are the polarization and magnetization of the material along the direction specified in the subscript, respectively. $E_{x, y, z}$ and $B_{x, y, z}$ are the electric and magnetic fields acting on the material along the directions in the subscript, respectively. $\epsilon_{0}$ and $\mu_{0}$ are the permittivity and permeability of the free space. $k_{0}$ and $\omega$ are the free-space wave vector and angular frequency of the incident wave. $\epsilon_{n m}$, $\mu_{n m}$, and $\kappa_{n m}$ are components in the relative permittivity, permeability, and chirality tensors of the material, where $n$ specifies the direction in which the exciting field acts, and $m$ is the direction along which the material response is as defined in Eqs. (7)-(10) in the text. 
[1] W. Francote and E. Linder (eds.), Chirality in Drug Research (Wiley/VCH, Weinheim, Germany, 2006).

[2] E. Hendry, T. Carpy, J. Johnston, M. Popland, R. V. Mikhaylovskiy, A. J. Lapthorn, S. M. Kelly, L. D. Barron, N. Gadegaard, and M. Kadodwala, Nat. Nanotechnol. 5, 783 (2010).

[3] T. J. Davis and D. E. Gómez, Phys. Rev. B 90, 235424 (2014).

[4] Y. Zhao, A. N. Askarpour, L. Sun, J. Shi, X. Li, and A. Alù, Nat. Commun. 8, 14180 (2017).

[5] W. Ma, H. Kuang, L. Xu, L. Ding, C. Xu, L. Wang, and N. A. Kotov, Nat. Commun. 4, 2689 (2013).

[6] Y. Tang and A. E. Cohen, Science (NY) 332, 333 (2011).

[7] R. Tullius, A. S. Karimullah, M. Rodier, B. Fitzpatrick, N. Gadegaard, L. D. Barron, V. M. Rotello, G. Cooke, A. Lapthorn, and M. Kadodwala, J. Am. Chem. Soc. 137, 8380 (2015). .

[8] E. Gorecka, M. Čepič, J. Mieczkowski, M. Nakata, H. Takezoe, and B. Žekš, Phys. Rev. E 67, 061704 (2003).

[9] N. Meinzer, E. Hendry, and W. L. Barnes, Phys. Rev. B 88, 041407 (2013).

[10] K. Bliokh, F. Rodríguez-Fortuño, F. Nori, and A. Zayats, Nat. Photon. 9, 796 (2015).

[11] K. Y. Bliokh, A. Y. Bekshaev, and F. Nori, Nat. Commun. 5, 3300 (2014).

[12] K. Y. Bliokh, M. A. Alonso, E. A. Ostrovskaya, and A. Aiello, Phys. Rev. A 82, 063825 (2010).

[13] M. M. Coles and D. L. Andrews, Phys. Rev. A 85, 063810 (2012).

[14] L. V. Poulikakos, P. Gutsche, K. M. McPeak, S. Burger, J. Niegemann, C. Hafner, and D. J. Norris, ACS Photon. 3, 1619 (2016).

[15] D. L. Andrews and M. M. Coles, Proc. SPIE 8274, 827405 (2012).

[16] L. Barron, Mol. Phys. 21, 241 (1971).

[17] K. Y. Bliokh and F. Nori, Phys. Rev. A 83, 021803(R) (2011).
[18] J. S. Choi and M. Cho, Phys. Rev. A 86, 1 (2012).

[19] M. Finazzi, P. Biagioni, M. Celebrano, and L. Duò, Phys. Rev. B 91, 195427 (2015).

[20] S. M. Barnett, L. Allen, R. P. Cameron, C. R. Gilson, M. J. Padgett, F. C. Speirits, and A. M. Yao, J. Opt. 18, 064004 (2016).

[21] E. Hendry, R. V. Mikhaylovskiy, L. D. Barron, M. Kadodwala, and T. J. Davis, Nano Lett. 12, 3640 (2012).

[22] L. D. Barron, Bio Syst. 20, 7 (1987).

[23] N. Yang and A. E. Cohen, J. Phys. Chem. B 115, 5304 (2011).

[24] Y. Tang and A. E. Cohen, Phys. Rev. Lett. 104, 163901 (2010).

[25] Z. Li, M. Mutlu, and E. Ozbay, J. Opt. 15, 023001 (2013).

[26] B. Wang, J.Zhou, T. Koschny, M. Kafesaki, and C. M. Soukoulis, J. Opt. A: Pure Appl. Opt. 11, 114003 (2009).

[27] C. Y. Chung and K. W. Whites, J. Electromagn. Waves. Appl. 10, 1363 (1996).

[28] K. H. Drexhage, J. Lumin. 1-2, 693 (1970).

[29] W. L. Barnes, J. Mod. Opt. 45, 661 (1998).

[30] E. Plum, Appl. Phys. Lett. 108, 241905 (2016).

[31] P. L. Stiles, J. A. Dieringer, N. C. Shah, and R. P. Van Duyne, Annu. Rev. Anal. Chem. 1, 601 (2008).

[32] Y. Liu, W. Zhao, Y. Ji, R.-Y. Wang, X. Wu, and X. D. Zhang, Europhys. Lett. 110, 17008 (2015).

[33] A. García-Etxarri and J. A. Dionne, Phys. Rev. B 87, 235409 (2013).

[34] M. V. Gorkunov, A. N. Darinskii, and A. V. Kondratov, J. Opt. Soc. Am. B 34, 315 (2017).

[35] F. Lu, Y. Tian, M. Liu, D. Su, H. Zhang, A. O. Govorov, and O. Gang, Nano Lett. 13, 3145 (2013).

[36] W. Zhang, T. Wu, R. Wang, and X. Zhang, J. Phys. Chem. C 121, 666 (2017).

[37] M. L. Nesterov, X. Yin, M. Schäferling, H. Giessen, and T. Weiss, ACS Photonics 3, 578 (2016).

[38] https://doi.org/10.24378/exe.244. 